Research Article

\title{
Investigation of Effects of Thermal Radiation, Magnetic Field, Eckert Number, and Thermal Slip on MHD Hiemenz Flow by Optimal Homotopy Asymptotic Method
}

\author{
Solomon Bati Kejela $\mathbb{D}^{D}$, Mitiku Daba $\mathbb{D}^{D}$, and Abebe Girum \\ Jimma University, College of Natural Sciences, Mathematics Department, Jimma, Ethiopia \\ Correspondence should be addressed to Solomon Bati Kejela; solomonbati@yahoo.com
}

Received 31 January 2021; Revised 24 March 2021; Accepted 29 March 2021; Published 12 April 2021

Academic Editor: Niansheng Tang

Copyright (C) 2021 Solomon Bati Kejela et al. This is an open access article distributed under the Creative Commons Attribution License, which permits unrestricted use, distribution, and reproduction in any medium, provided the original work is properly cited.

\begin{abstract}
Analytical investigation of thermal radiation, Prandtl number, Eckert number, permeability parameter, magnetic field, velocity, and thermal slip effects on magnetohydrodynamic Hiemenz flow over a permeable plate with forced convection has been presented. Similarity variable conversion method has been applied to transmute the fundamental governing equations of the fluid dynamics in flow into a pair of nonlinear third-order ordinary differential equations and is analytically solved by the optimal homotopy asymptotic method (OHAM). The influences of several relevant physical parameters in the model on velocity and temperature of the fluid have been studied and analysed profoundly by use of graphs and tables. It is detected that, with mounting value of suction/blowing parameter and magnetic field parameter, the skin friction coefficient enhances. Likewise, it is seen that the Nusselt number increases with enhancing value of magnetic parameter. It is also witnessed that the velocity increases as the Eckert number, blowing/suction parameter, and permeability parameter increase, but it decays against magnetic field and velocity slip parameter. Moreover, the result reveals that the fluid temperature upsurges along with snowballing the radiant heat, magnetic field parameter, and the Eckert number. However, it descends against thermal slip parameter, Prandtl number, wall temperature exponent, and velocity slip parameter. A comparison with previous studies has been made, and the result shows an excellent agreement.
\end{abstract}

\section{Introduction}

The study of Hiemenz (stagnation-point) flow plays an important role in the study of plentiful natural and industrial phenomena because of its applications in discovering flows over the tips of submarines, the tip of ships, and aircraft. It is also vital in various engineering disciplines like hydrodynamic processes, refrigeration of electronic materials using fans, freezing of nuclear apparatus, and so forth. The theoretical inquiry of the tide of Newtonian fluid near a two-dimensional stop off-point flow in the route of a motionless semi-infinite barricade has been studied before all by Hiemenz [1]. Hiemenz applied a similarity variable conversion method to reduce the Navier Stokes equations to equivalent solvable nonlinear ODEs. The study of magnetohydrodynamic (MHD) boundary layer flow on a continuous stretching sheet has numerous applications in industrial manufacturing processes such as the aerodynamic extrusion of plastic sheets, wire drawing, glass fiber and paper production, liquid film, hot rolling, drawing of plastic films, metal, polymer extrusion, and metal spinning. The MHD stagnation-point flow problems have theoretical and practical applications in manufacturing processes like boundary layer along material handling conveyers, blood flow problems, extrusion of plastic sheets, geothermal energy extractions, cooling of infinite metallic plate in cooling bath, petroleum industries, and so forth; due to this, they have attracted the attention of many researchers. Ishak et al. [2] discussed the MHD stagnation-point flow towards a stretching surface with variable surface temperature, whereas the MHD effect past a shrinking sheet has been analysed by Mahapatra et al. [3]. Since then, several studies 
have been conducted on stretching/shrinking problems [4-12].

The effects of thermal radiation and heat transfer play an essential role in the fluid flow problem. The modern system of electric power generation, plasma, space vehicles, astrophysical flows, and cooling of nuclear reactors are governed by applications of thermal radiation and heat transfer of fluid flow. Because of its importance, the impact of thermal radiation, thermal slip, and heat transfer on MHD stagnation-point flow for different geometrical configurations has been tested by numerous researchers [13-19]. Makinde [20] investigated the hydromagnetic varied convection Hiemenz flow towards upright plate entrenched in an extremely permeable medium with radiation and internal heat generation. He discussed some aspects of the pertinent parameters on the dimensionless axial velocity, temperature and the concentration profiles, local skin friction, and local Nusselt number. Wubishet [21] examined the effect of induced magnetic field on MHD Hiemenz flow and heat transfer due to upper-convicted Maxwell fluid over a stretching sheet in the company of nanoparticles which is heated convectively. Numerical results are obtained for velocity, temperature, concentration profiles, skin friction coefficient, local Nusselt number, and Sherwood number. His result indicated that skin friction coefficient, the local Nusselt number, and Sherwood number increase with an increase in velocity ratio and Deborah number and decrease as the values of magnetic field increase.

An analysis for the magnetohydrodynamic (MHD) axisymmetric Hiemenz flow and heat transmission over a shrinking sheet was presented by Mahapatra and Nandy [22], in the paper, the temperature profiles are obtained for the sheet with prescribed surface temperature and the sheet with prescribed surface heat flux. The effects of various physical parameters on Nusselt number and skin friction coefficient on the flow and heat transfer characteristics are discussed. Khan et al. [23] have investigated the effects of homogeneous-heterogeneous reactions in MHD flow of Casson fluid flow over a stretched surface and they discussed the behavior of velocity, temperature, concentration, drag force, and heat transfer rate. They found that, with larger magnetic parameter, the velocity field decreases, whereas surface drag force enhances. Temperature profile and heat transfer rate decay for higher estimation of Prandtl number. Shateyi and Fazle [24] analysed the problem of MHD mixedconvection stagnation-point flow towards a nonlinearly stretching vertical sheet in the presence of thermal radiation and viscous dissipation. It is noticed that the thermal boundary layer thicknesses and velocity are decreasing against the value of the nonlinearity parameter. The effect of injection on the MHD mixed-slip flow is to enhance the velocity field.

Khan and Faris [25] developed Cattaneo-Christov doublediffusion model with nanofluid for Williamson fluid and constructed convergent solution for the model. In this research work, they discussed the physical features of MHD stagnation-point flow of Williamson nanomaterial over a stretched surface. Their results showed velocity boosts up significantly via velocity ratio parameter and variation of
Weissenberg number yields improvement of temperature. The effect of the magnetic field, the radiation parameter, the uniform suction/injection parameter, and Hall parameter on MHD flow of a viscous, Newtonian, and electrically conducting fluid past a porous rotating infinite disk in consequence of Hall effect was studied by Devi et al. [26]. A comprehensive study on entropy generation in nanofluid flow towards a curved stretched surface with combined effects of activation energy, Brownian motion, viscous dissipation, nonlinear mixed convection, MHD, Joule heating, and thermophoresis diffusion is presented by Khan and Faris [27]. It is reported that velocity field is an increasing function of curvature parameter, while opposite impact is observed for Deborah number and velocity slip parameter. Nusselt number is increased via larger Eckert number and declines against thermophoretic parameter.

Khan and Faris [28] presented mathematical modeling and numerical simulation for the steady, incompressible two-dimensional Darcy-Forchheimer nanofluid flow of viscous material towards a stretched surface and discussed the behavior of pertinent flow parameters on the velocity, temperature, Bejan number, and entropy generation for both nanoparticles for both nanoparticles (silicon dioxide and molybdenum). The effect of entropy generation in flow of viscous fluid of hybrid nanoparticles over a stretchable rotating disk subject to nonlinear thermal radiation and slip conditions is analysed by Khan [29]. The impacts of mixedconvection parameter, porosity parameter, velocities as well as thermal slips parameters, stretching parameter, nonlinear radiation parameter, and Reynolds number on radial velocity profile, tangential velocity profile, and temperature profile are studied.

Fluids showing slip are important in the areas of technology and industry such as in the polishing of artificial heart valves and internal cavities. Fang et al. [30] analytically solved the MHD flow under slip conditions over a permeable shrinking surface, and they reported that the velocity slip at the shrinking surface greatly affects the velocity distribution and drag forces on the wall. El-Aziz and Ahmed [31] numerically analysed the influences of slip velocity and induced magnetic field on MHD stagnation-point flow and heat transfer of Casson fluid over a stretching sheet. Their result revealed that, with an increase in slip parameter, the velocity distribution reduces, whereas the temperature profile enhances.

However, to the best of the authors' knowledge, no study has been previously reported on the problem of MHD Hiemenz boundary layer flow at stagnation region against flat plate through a holey medium with thermal radiation, induced magnetic field, and velocity and thermal slip effect. In view of this and importance of MHD stagnation-point flow in engineering and in various technological applications and in numerous production processes such as the aerodynamic squeezing of polymers, rolling at high temperature, cooling control technology, and glass fibber production, the authors in the present paper aim to examine the effect of thermal radiation, magnetic field, Eckert number, Prandtl number, penetrability parameter, velocity, and thermal slip parameters on MHD Hiemenz flow at stagnation region 
against flat plate over a porous medium with thermal radiation and slip effect. The governing equations and their associated boundary conditions are at first changed into dimensionless form with the help of similarity variables; then the resulting system of nonlinear high-order ordinary differential equations is solved by the optimal homotopy asymptotic method (OHAM). The results obtained are then compared with those from the available literature for some particular values of the physical parameters, and it is found that they are in an excellent agreement. Graphical results for the velocity and temperature of the flow are discussed.

\section{Basic Principles of Optimal Homotopy Asymptotic Method}

The OHAM is an amendment of the homotopy asymptotic method (HAM), which is based on reducing the residual error. In this proposed method, the rheostat and adjustment of the confluence region are set properly. To simply illustrate the underlying rules of this method as expanded by Marinca and Herisanu [32] and different scholars, we take the nonlinear DE expressed as

$$
L(v(x))+r(x)+N(v(x))=0, \quad x \in \Omega,
$$

with boundary condition:

$$
B\left(x, \frac{\mathrm{d} v}{\mathrm{~d} x}\right)=0,
$$

where $\Omega$ is problem domain, $L$ and $\mathrm{N}$ represent the linear and nonlinear operators, $v(x)$ is an unknown function, and $r(x)$ is a known function. The optimal homotopy equation (also called deformation equation) is constructed as

$$
(1-p)[L(\phi(x ; p))+a(x)]=h(p)[L(\phi(x ; p))+a(x)+N(\phi(x ; p))+a(x)],
$$

where $0 \leq p \leq 1$ is an inserting parameter.

$h(p)=\sum_{k=1}^{m} p^{k} c_{k}$ is an auxiliary function that determines convergence of a problem's solution. The auxiliary function $h(p)$ helps to adjust the convergence domain in addition to controlling a convergence region. By the suggested method, we will achieve approximate solution in a series form whenever the function $\phi\left(x ; p, c_{j}\right)$ is expressed in Taylor's series about $p$.

$$
\phi\left(x ; p, c_{j}\right)=v_{0}(x)+\sum_{k=1}^{\infty} v_{k}\left(x, c_{j}\right) p^{k}, \quad j=1,2,3, \ldots
$$

As it is identified by many scholars, the convergence of equation (4) depends upon the values of convergence parameters $c_{j}(j=1,2,3, \ldots, m)$. If the series in equation (4) is convergent, then

$$
\widetilde{v}=v_{0}(x)+\sum_{k=1}^{m} v_{k}\left(x ; c_{j}\right) .
$$

Plugging equation (5) in equation (1) results in the following residual:

$$
R\left(x ; c_{j}\right)=L\left(\widetilde{v}\left(x ; c_{j}\right)\right)+r(x)+N\left(\widetilde{v}\left(x ; c_{j}\right)\right) .
$$

If the function in equation (6) will vanish or equals zero, then $\widetilde{v}$ will be an accurate solution of the given problem but this is not usually the case in nonlinear problems. The values of $c_{j}(j=1,2,3, \ldots, m)$ can be optimally determined via various methods like colocation method, least-square method, Galerkin's method, Ritz method, the Kantorovich method, and so forth, which can be utilized. Finally, substituting the values of these convergence control parameters in equation (5), one can get the analytical approximate solution of a problem. In our case, we applied the least-square method, because it is a powerful method to find optimal values of convergence control parameter presented in a solution of a problem in using different numerical and analytical methods [33].

\section{Mathematical Formulation}

In developing a model that describes the flow under consideration, we consider a two-dimensional steady laminar enforced convection magnetohydrodynamic Hiemenz flow at the stagnation area against a smooth plate through a holey medium with radiant heat, where a plate is placed aligned to the $x$-axis and the $y$-axis is ordinary to the sheet. The liquid in flow is assumed to be electrically conducted and incompressible. A continuous magnetic field of strength $B_{0}$ is applied along the $y$ direction, the wall temperature has been taken as variable, and the surface mass fluctuation is supposed to be uniform. The induced magnetic field, the Hall effects, and the viscus dissipation terms are neglected. The schematic diagram representing the physical phenomena of the problem is illustrated in Figure 1. Following the Raptis and Takhar [34] model designed for the permeable medium and by prefacing the boundary layer approximation, the governing equations describing the flow are as follows:

Continuity:

$$
\frac{\partial u}{\partial x}+\frac{\partial v}{\partial y}=0
$$

Momentum:

$$
u \frac{\partial u}{\partial x}+v \frac{\partial u}{\partial y}=\frac{-1}{\rho} \frac{\partial P}{\partial x}+v \frac{\partial^{2} u}{\partial y^{2}}-\frac{v}{K} u-\frac{\sigma B_{0}^{2}}{\rho} u .
$$

Energy:

$$
u \frac{\partial T}{\partial x}+v \frac{\partial T}{\partial y}=\alpha \frac{\partial^{2} T}{\partial y^{2}}-\frac{1}{\rho c_{p}} \frac{\partial q_{r}}{\partial y}+v \frac{1}{\rho c_{p}}\left(\frac{\partial u}{\partial y}\right)^{2},
$$




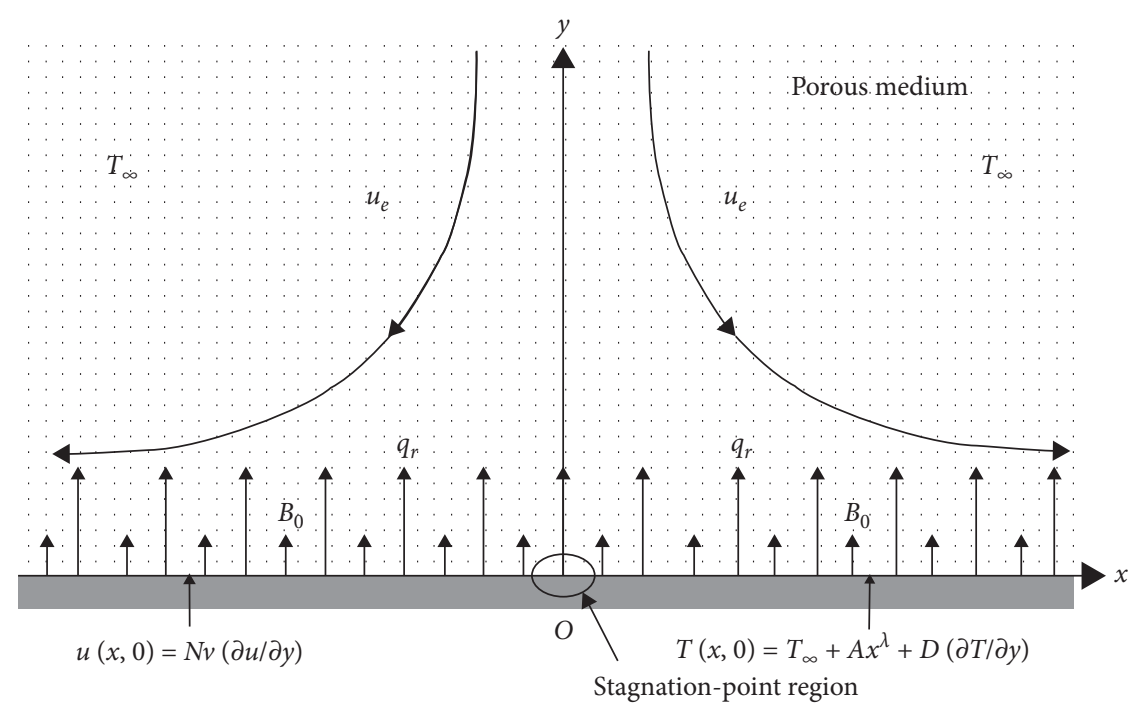

Figure 1: Geometry of the flow problem.

where $u$ and $v$ are, respectively, the velocity constituents on the way to the $\mathrm{x}$ - and $\mathrm{y}$-directions, $P$ is the pressure, $\rho$ represents density, $K$ is porosity parameter, $\mu$ is the fluid viscosity coefficient, $v=(\mu / \rho)$ is the kinematic viscosity, $\sigma$ is the electrical conductivity, $T$ is a fluid temperature and a permeable medium which are in local thermal equilibrium, $\alpha=\left(k / \rho c_{p}\right)$ is the equivalent thermal diffusivity, where $k$ is a heat conduction coefficient, $c_{p}$ is a specific heat at unchanged pressure, and $q_{r}$ represents the radiative heat flux.

The boundary conditions of the problems are as follows:

$$
\begin{aligned}
& v=v_{w}, u=u_{w}+u_{s l i p}=0+N v \frac{\partial u}{\partial y}, \\
& \left.T=T_{w}+T_{\text {slip }}=T_{\infty}+A x^{\lambda}+D \frac{\partial T}{\partial y},\right\}, \quad \text { at } y=0, \\
& \left.\begin{array}{l}
u=u_{l}=a x, \\
T=T_{\infty}
\end{array}\right\}, \quad \text { at } y \longrightarrow \infty,
\end{aligned}
$$

where $v_{w}$ is an invariable surface mass flux, which is positive for blowing and negative for suction; $u_{\text {slip }}$ is velocity slip, which is comparative with a shear stress in the layer of fluid next to wall of a plate and is given by $N v(\partial u / \partial y)$, where $\mathrm{N}$ is Navier's constant slip length; $\lambda$ is the exponent of a wall temperature; $D$ is temperature slip factor, and $u_{l}=m x$ is the unrestricted flow velocity where $m$ denotes every positive number.

Using the equation $u_{l}=m x$ in (8), the equation becomes reduced to

$$
u_{l} \frac{\mathrm{d} u_{l}}{\mathrm{~d} x}=\frac{-1}{\rho} \frac{\partial P}{\partial x}-\frac{v}{K} u_{l}-\frac{\sigma B_{0}^{2}}{\rho} u_{l} .
$$

From equation (12), we have

$$
\frac{-1}{\rho} \frac{\partial P}{\partial x}=u_{l} \frac{\mathrm{d} u_{l}}{\mathrm{~d} x}+\frac{v}{K} u_{l}+\frac{\sigma B_{0}^{2}}{\rho} u_{l} .
$$

Upon using equation (13) in equation (8), we obtain

$$
u \frac{\partial u}{\partial x}+v \frac{\partial u}{\partial y}=u_{l} \frac{\mathrm{d} u_{l}}{\mathrm{~d} x}+\frac{v}{K}\left(u_{l}-u\right)+\frac{\sigma B_{0}^{2}}{\rho}\left(u_{l}-u\right)+v \frac{\partial^{2} u}{\partial y^{2}} .
$$

From (14), it is obvious that $u_{l}$ affects the fluid flow. Thus, convection of heat will be affected considerably, and this shows that the flow is a type of forced convection.

Using Rosseland approximation, we can define or express the heat energy transmitted by electromagnetic surfs in contrast to heat transmitted by conduction in terms of temperature as follows:

$$
q_{r}=\frac{-4 \sigma^{\prime}}{3 k_{1}} \frac{\partial T^{4}}{\partial y}
$$

where $\sigma^{\prime}$ represents the Stefan-Boltzmann constant and $k_{1}$ represents the mean immersion coefficient. Assuming that the temperature variances in the interior of the flow are appropriately small and expanding $T^{4}$ about the unrestricted stream temperature $T_{\infty}$ and disregarding higher-order expressions, we get

$$
T^{4} \approx 4 T_{\infty}^{3}-3 T_{\infty}^{4} \text {. }
$$

Upon using equation (16) in equation (15), we get

$$
\frac{\partial q_{r}}{\partial y}=-\frac{16 \sigma^{\prime} T_{\infty}^{3}}{3 k_{1}}\left(\frac{\partial^{2} T}{\partial y^{2}}\right)
$$

Substituting equation (17) into equation (9), we obtain

$$
u \frac{\partial T}{\partial x}+v \frac{\partial T}{\partial y}=\alpha \frac{\partial^{2} T}{\partial y^{2}}+\frac{16 \sigma^{\prime} T_{\infty}^{3}}{3 \rho c_{p} k_{1}}\left(\frac{\partial^{2} T}{\partial y^{2}}\right)+v \frac{1}{\rho c_{p}}\left(\frac{\partial u}{\partial y}\right)^{2},
$$

where $\alpha=\left(k / \rho c_{p}\right)$ is the thermal diffusivity, and this $s$ equation illustrates that radiation enhances thermal diffusivity. 
Let us define the stream function satisfying equation (7) as follows:

$$
\begin{aligned}
& u=\frac{\partial \phi}{\partial y}, \\
& v=-\frac{\partial \phi}{\partial x} .
\end{aligned}
$$

To solve equations (8) and (9), the following similarity variables have been introduced:

$$
\begin{aligned}
\eta & =y \sqrt{\left(\frac{a}{\alpha}\right)}, \\
\phi & =\sqrt{a \alpha} x f(\eta), \\
\theta(\eta) & =\frac{T-T_{\infty}}{T_{w}-T_{\infty}} .
\end{aligned}
$$

From equations (19) and (20), we get

$$
\begin{aligned}
& u=a x f^{\prime}(\eta), \\
& v=-\sqrt{a \alpha} f(\eta),
\end{aligned}
$$

where $f^{\prime}(\eta)=(\mathrm{d} f / \mathrm{d} \eta), T_{w}$ denotes a constant surface temperature, and $\theta(\eta)$ is a nondimensional form of the temperature.

Using equation (21) in equation (14), we obtain

$$
\operatorname{Pr} f^{\prime \prime \prime}+\left(1-f^{\prime 2}\right)+f f^{\prime \prime}+\left(\Omega+M^{2}\right)\left(1-f^{\prime}\right)=0 \text {. }
$$

We have the following boundary conditions:

$$
\begin{aligned}
f(0) & =s, \\
f^{\prime}(0) & =\beta f^{\prime \prime}(0), \quad \text { at } y=0, \\
f^{\prime}(\eta) & =1, \quad \text { as } \eta \longrightarrow \infty,
\end{aligned}
$$

where $\operatorname{Pr}=(v / \alpha)$ is the Prandtl number, $\Omega=(v / k a)$ is the permeability parameter, $M=\sqrt{\left(\sigma B_{0}^{2} / \rho a\right)}$ is the charismatic parameter, $s=-\left(v_{w} / \sqrt{a \alpha}\right)$ is the mass transfer parameter, and $\beta=N v \sqrt{a / \alpha}$ is the dimensionless velocity slip parameter.

From equations (10), (11), and (20), we have

$$
\begin{aligned}
& \frac{\partial T}{\partial y}=\theta^{\prime}\left(T_{w}-T_{\infty}\right) \sqrt{\frac{a}{\alpha}}, \\
& \frac{\partial^{2} T}{\partial y^{2}}=\frac{a}{\alpha} \theta^{\prime \prime}\left(T_{w}-T_{\infty}\right), \\
& \frac{\partial T}{\partial x}=A \lambda \theta x^{\lambda-1} .
\end{aligned}
$$

Plugging equation (24) into equation (18), we obtain

$$
\left(1+\frac{4 R}{3}\right) \theta^{\prime \prime}+f \theta^{\prime}-\lambda f^{\prime} \theta+\operatorname{PrEc}\left(f^{\prime \prime}\right)^{2}=0 .
$$

The above equation is subject to the following boundary conditions:

$$
\begin{aligned}
& \theta(0)=1+\gamma \theta^{\prime}(0), \\
& \theta(\eta)=0, \quad \text { as } \eta \longrightarrow \infty
\end{aligned}
$$

where $\gamma=D \sqrt{a / \alpha}$ is dimensionless thermal slip parameter, $R=\left(4 \sigma^{\bullet} T_{\infty}^{3} / k_{1} k\right)$ is radiation parameter, and $\mathrm{Ec}=\left(u_{l}^{2} / c_{p}\left(T_{w}-T_{\infty}\right)\right)$ is the Eckert number.

\section{Solution of the Problem Using the OHAM}

Applying OHAM on the nonlinear ODEs equations (22) and (25), we construct the following equations:

$$
\left.\begin{array}{l}
(1-p)\left(f^{\prime \prime \prime}\right)-H_{1}(p)\left[\operatorname{Pr} f^{\prime \prime \prime}+\left(1-f^{\prime 2}\right)+f f^{\prime \prime}+\left(\Omega+M^{2}\right)\left(1-f^{\prime}\right)=0\right]=0 \\
(1-p)\left(\theta^{\prime \prime}\right)-H_{2}(p)\left[\left(1+\frac{4 R}{3}\right) \theta^{\prime \prime}+f \theta^{\prime}-\lambda f^{\prime} \theta+\operatorname{PrEc}\left(f^{\prime \prime}\right)^{2}\right]=0
\end{array}\right\},
$$

where $p \in[0,1]$ and $f, \theta, H_{1}(p), H_{2}(p)$ are expanded as follows:

$$
\begin{aligned}
f & =f_{0}+p f_{1}+p^{2} f_{2}+p^{3} f_{3}, \\
\theta & =\theta_{0}+p \theta_{1}+p^{2} \theta_{2}+p^{3} \theta_{3}, \\
H_{1}(p) & =p c_{1}+p^{2} c_{2}+p^{3} c_{3}, \\
H_{2}(p) & =p c_{4}+p^{2} c_{5}+p^{3} c_{6} .
\end{aligned}
$$

Using equation (28) in equation (27) and simplifying, rearranging, and collecting terms with common powers of $p$, we get the following zeroth-, first-, second-, and third-order problems together with their boundary conditions.

The zeroth-order problem is

$$
\left\{\begin{array}{l}
f^{\prime \prime \prime}(\eta)=0 \\
\theta^{\prime \prime}(\eta)=0
\end{array}\right.
$$

with boundary conditions:

$$
\begin{aligned}
f_{0}(0) & =s, \\
f_{0}^{\prime}(0) & =\beta f_{0}^{\prime \prime}(0), \\
f_{0}^{\prime}(\eta)=1, \quad \text { as } \eta \longrightarrow \infty, & \\
\theta_{0}(0) & =1+\gamma \theta_{0}^{\prime}(0), \\
\theta_{0}(\eta) & =0, \quad \text { as } \eta \longrightarrow \infty .
\end{aligned}
$$

The solution for equation (29) with BCs (30) is 


$$
\left\{\begin{array}{l}
f_{0}(\eta)=\frac{1}{20}\left(1-\beta f_{0}^{\prime \prime}(0)\right) \eta^{2}+\beta f_{0}^{\prime \prime}(0) \eta+s \\
\theta_{0}(\eta)=\left(1+\gamma \theta_{0}^{\prime}(0)\right)\left(1-\frac{\eta}{10}\right)
\end{array}\right.
$$

The first-order problem is

$$
\left\{\begin{array}{l}
f_{1}^{\prime \prime}\left(\eta, c_{1}\right)=\frac{c_{1}}{\operatorname{Pr}}\left(1-f_{0}^{\prime 2}+f_{0} f_{0}^{\prime \prime}+\left(\Omega+M^{2}\right)\left(1-f_{0}^{\prime}\right)\right), \\
\theta_{1}^{\prime \prime}\left(\eta, c_{4}\right)=\frac{3 c_{4}}{3+4 R}\left(f_{0} \theta_{0}^{\prime}-\lambda f_{0}^{\prime} \theta_{0}+\operatorname{PrEc}\left(f_{0}^{\prime \prime}\right)^{2}\right),
\end{array}\right.
$$

with boundary conditions:

$$
\left\{\begin{array}{l}
f_{1}(0)=0, \\
f_{1}^{\prime}(0)=0, \\
\theta_{1}(0)=0, \\
f_{1}^{\prime}(\eta)=0, \\
\theta_{1}(\eta)=0, \quad \text { as } \eta \longrightarrow \infty .
\end{array}\right.
$$

Its solution is

$$
\begin{aligned}
f_{1}\left(\eta, c_{1}\right)=\frac{5 c_{1}}{\operatorname{Pr}}\left[\begin{array}{c}
\left(1+\Omega+M^{2}\right)\left(1-\beta f_{0}^{\prime \prime}(0)\right)+\left(\frac{s}{10}-\beta f_{0}^{\prime \prime}(0)\right)\left(\frac{\eta^{3}}{30}-\frac{\eta^{2}}{2}\right) \\
+\frac{1}{6}\left[\beta f_{0}^{\prime \prime}(0)\left(\beta f_{0}^{\prime \prime}(0)-1\right)+\left(\Omega+M^{2}\right) f_{0}^{\prime \prime}(0)-\frac{1}{10}\right]\left(\frac{\eta^{4}}{200}-\eta^{2}\right) \\
+\frac{1}{6}\left[\beta f_{0}^{\prime \prime}(0)\left(1-\frac{\beta f_{0}^{\prime \prime}(0)}{2}\right)-\frac{1}{2}\right]\left(\frac{\eta^{5}}{5000}-\frac{\eta^{2}}{2}\right)
\end{array}\right], \\
\theta_{1}\left(\eta, c_{4}\right)=
\end{aligned}
$$

The second-order problem is

$$
\left\{\begin{array}{l}
f_{2}^{\prime \prime}\left(\eta, c_{1}, c_{2}\right)=\left(c_{1}+c_{2}\right) f_{1}^{\prime \prime}+\frac{c_{2}}{\operatorname{Pr}}\left[1-f_{0}^{\prime 2}+f_{0} f_{0}^{\prime \prime}+\left(\Omega+M^{2}\right)\left(1-f_{0}^{\prime}\right)+f_{0} f_{1}^{\prime \prime}+f_{1} f_{0}^{\prime \prime}-2 f_{0}^{\prime} f_{1}^{\prime}-f_{1}\right] \\
\theta_{2}^{\prime \prime}\left(\eta, c_{4}, c_{5}\right)=\left(1+c_{4}\right) \theta_{1}^{\prime \prime}+\frac{3 c_{4}}{3+4 R}\left[f_{1} \theta_{0}^{\prime}+f_{0} \theta_{1}^{\prime}-\lambda\left(f_{1}^{\prime} \theta_{0}+f_{0}^{\prime} \theta_{1}\right)+2 \operatorname{PrEc} f_{0}^{\prime \prime} f_{1}^{\prime \prime}\right] \\
+\frac{3 c_{5}}{3+4 R}\left(f_{0} \theta_{0}^{\prime}-\lambda f_{0}^{\prime} \theta_{0}+\operatorname{PrEc} f_{0}^{\prime \prime 2}\right) .
\end{array}\right.
$$

The above problem is subject to the following boundary

$$
\left\{\begin{array}{l}
f_{2}(0)=0, \\
f_{2}^{\prime}(0)=0, \\
\theta_{2}(0)=0, \\
f_{2}^{\prime}(0)=0, \\
\theta_{2}(0)=0, \quad \text { as } \eta \longrightarrow \infty .
\end{array}\right.
$$

The solution for this second-order problem is 


$$
\begin{aligned}
& f_{2}\left(\eta, c_{1}, c_{2}\right)=\frac{10}{\operatorname{Pr}}\left[\begin{array}{c}
\left(1+\left(\Omega+M^{2}\right)\left(1-\beta f_{0}^{\prime \prime}(0)\right)+\frac{s}{10}-\beta f_{0}^{\prime \prime}(0)\left(\frac{s}{10}+\beta f_{0}^{\prime \prime}(0)\right)\right)\left(\left(c_{1}+c_{1}^{2}\right)\left(\frac{\eta^{3}}{6}-\frac{\eta^{2}}{4}\right)\right) \\
+\frac{c_{1}^{2}}{\operatorname{Pr}}\left(\frac{\eta^{4}}{240}-\frac{\eta^{3}}{12}+\frac{5 \eta^{2}}{12}+\left(1-\beta f_{0}^{\prime \prime}(0)\right)\left(\frac{-\eta^{6}}{14400}+\frac{\eta^{5}}{800}+\frac{25 \eta^{2}}{24}\right)-\left(2 \beta f_{0}^{\prime \prime}(0)+1\right)\left(\frac{\eta^{5}}{1200}-\frac{\eta^{4}}{480}+\frac{25 \eta^{2}}{12}\right)\right) \\
\left(\beta f_{0}^{\prime \prime}(0)\left(\beta f_{0}^{\prime \prime}(0)-1\right)+\left(\Omega+M^{2}\right)\left(\beta f_{0}^{\prime \prime}(0)-\frac{1}{10}\right)\right)\left(c_{1}+c_{1}^{2}\right)\left(\frac{\eta^{4}}{2400}-\frac{\eta^{2}}{12}\right)+\frac{c_{1}^{2}}{\operatorname{Pr}}\left(\frac{\eta^{5}}{1200}-\frac{250 \eta^{2}}{9}+\frac{50 \eta^{2}}{19}\right) \\
+\frac{c_{1}^{2}}{\operatorname{Pr}}\left(\left(1-\beta f_{0}^{\prime \prime}(0)\right)\left(\frac{-\eta^{7}}{720000}+\frac{\eta^{5}}{2400}-\frac{65 \eta^{2}}{72}\right)-\left(2 \beta f_{0}^{\prime \prime}(0)+1\right)\left(\frac{\eta^{6}}{7200}-\frac{\eta^{4}}{1440}+\frac{35 \eta^{2}}{36}\right)\right)
\end{array}\right] \\
& +\frac{10}{\operatorname{Pr}}\left[\begin{array}{c}
\left(c_{1}+c_{1}^{2}+c_{2}\right)\left(\frac{\eta^{5}}{60000}-\frac{\eta^{2}}{24}\right) \\
\left(\beta f_{0}^{\prime \prime}(0)\left(1-\frac{\beta f_{0}^{\prime \prime}(0)}{2}\right)-\frac{1}{2}\right)\left(+\frac{c_{1}^{2}}{\operatorname{Pr}}\left(\begin{array}{c}
\frac{\eta^{5}}{360000}-\frac{\eta^{4}}{72}+\frac{\eta^{3}}{480}+\frac{25 \eta^{2}}{12} \\
+\frac{c_{1}^{2}}{\operatorname{Pr}}\left(\beta f_{0}^{\prime \prime}(0)\left(\beta f_{0}^{\prime \prime}(0)-2\right)\right)\left(\left(\beta f_{0}^{\prime \prime}(0)+\frac{1}{2}\right)\left(\frac{\eta^{7}}{520000}-\frac{\eta^{4}}{288}+\frac{5 \eta^{2}}{9}\right)+\left(1-\beta f_{0}^{\prime \prime}(0)\right)\left(\frac{\eta^{8}}{40320}-\frac{\eta^{5}}{7200}+\frac{125 \eta^{2}}{504}\right)\right) \\
\left.+\frac{\eta^{8}}{201600000}-\frac{\eta^{5}}{14400}+\frac{310 \eta^{2}}{2016}\right)
\end{array}\right)\right. \\
+\left(1+\Omega+M^{2}\right)\left(\frac{\eta^{3}}{60}-\frac{\eta^{2}}{4}\right)+\left(10 \beta f_{0}^{\prime \prime}(0)\left(\beta f_{0}^{\prime \prime}(0)-1\right)+\left(\Omega+M^{2}\right)\left(\beta f_{0}^{\prime \prime}(0)-1\right)\right)\left(\frac{\eta^{4}}{2400}-\frac{\eta^{2}}{12}\right) \\
+\left(s-\beta f_{0}^{\prime \prime}(0)\left(s+10 \beta f_{0}^{\prime \prime}(0)\right)-10 \beta f_{0}^{\prime \prime}(0)\left(\Omega+M^{2}\right)\right)\left(\frac{\eta^{3}}{600}-\frac{\eta^{2}}{40}\right)
\end{array}\right]
\end{aligned}
$$

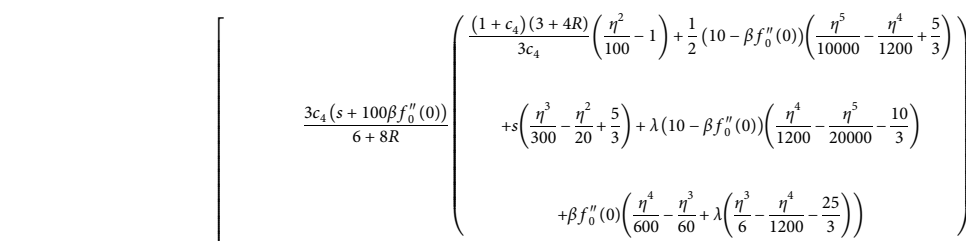

$$
\begin{aligned}
& \theta_{2}\left(\eta, c_{4}, c_{5}\right)=\frac{-3 c_{4}\left(1+\gamma \theta_{0}^{\prime}\right)}{3+4 R}\left(\begin{array}{c}
\frac{\left(1+c_{4}\right)(3+4 R)}{3 c_{4}}\left(\frac{\eta^{3}}{30}-\frac{100}{3}\right)+\frac{1}{2}\left(10-\beta f_{0}^{\prime \prime}(0)\right)\left(\frac{\eta^{6}}{3000}-\frac{\eta^{4}}{36}+\frac{500}{9}\right) \\
6+8 R \\
+\beta f_{0}^{\prime \prime}(0)\left(\frac{\eta^{5}}{200}-\frac{5 \eta^{3}}{9}+\frac{500}{9}\right) \\
+s\left(\frac{\eta^{4}}{12000}-\frac{5 \eta^{2}}{3}+\frac{250}{3}\right)+\lambda\left(1-\beta f_{0}^{\prime \prime}(0)\right)\left(\frac{\eta^{4}}{36}-\frac{\eta^{6}}{9000}-\frac{500}{3}\right) \\
+\lambda\left(\frac{5 \eta^{3}}{9}-\frac{\eta^{5}}{600}-\frac{3500}{9}\right)
\end{array}\right) \\
& \frac{3 c_{4}\left(1-\beta f_{0}^{\prime \prime}(0)\right)+2 \lambda\left(\beta f_{0}^{\prime \prime}(0)-1\right)}{3+4 R}\left(\begin{array}{c}
\frac{\left(1+c_{4}\right)(3+4 R)}{3 c_{4}}\left(\frac{\eta^{4}}{600}-\frac{50}{3}\right)+\frac{1}{36}\left(10-\beta f_{0}^{\prime \prime}(0)\right)\left(\frac{\eta^{6}}{3000}-\frac{\eta^{4}}{36}+\frac{500}{9}\right) \\
+\beta f_{0}^{\prime \prime}(0)\left(\frac{\eta^{7}}{3500}-\frac{\eta^{4}}{4}+\frac{2500}{7}+\frac{\eta^{6}}{4500}-\frac{5 \eta^{3}}{18}\right)+\frac{s}{6}\left(\frac{\eta^{5}}{500}-5 \eta^{2}\right) \\
+\frac{\lambda}{18}\left(1-\beta f_{0}^{\prime \prime}(0)\right)\left(\frac{\eta^{4}}{4}-\frac{\eta^{7}}{14000}-\frac{12500}{7}\right)+\frac{\lambda \beta f_{0}^{\prime \prime}(0)}{6}\left(\frac{5 \eta^{3}}{3}-\frac{\eta^{6}}{3000}-\frac{4000}{3}\right)
\end{array}\right) \\
& \frac{-3 c_{4}\left(1+\gamma \theta_{0}^{\prime}\right)}{3+4 R}\left[\begin{array}{c}
\operatorname{Pr}\left(\begin{array}{c}
\left(1+\left(\Omega+M^{2}\right)\left(1-\beta f_{0}^{\prime \prime}(0)\right)+\frac{s}{10}-\frac{\beta f_{0}^{\prime \prime}(0)}{10}\left(s+10 \beta f_{0}^{\prime \prime}(0)\right)\right)\left(\frac{\eta^{3}}{1200}-\frac{\eta^{4}}{48}+125\right)+\lambda\left(\frac{\eta^{4}}{12}-\frac{5 \eta^{3}}{6}-\frac{\eta^{5}}{400}+125\right) \\
+\left(\left(\beta f_{0}^{\prime \prime}(0)\right)^{2}-\beta f_{0}^{\prime \prime}(0)+\left(\Omega+M^{2}\right)\left(\beta f_{0}^{\prime \prime}(0)\right)-\frac{1}{10}\right)\left(\frac{\eta^{6}}{7200}-\frac{\eta^{4}}{144}+\frac{500}{9}\right)+\lambda\left(\frac{\eta^{5}}{1200}-\frac{5 \eta^{3}}{18}+\frac{\eta^{4}}{72}-\frac{\eta^{6}}{18000}+\frac{1000}{9}\right) \\
+\left(\beta f_{0}^{\prime \prime}(0)-\frac{1}{2}\left(\beta f_{0}^{\prime \prime}(0)\right)^{2}-\frac{1}{2}\right)\left(\frac{\eta^{7}}{2520000}-\frac{\eta^{4}}{288}+\lambda\left(\frac{\eta^{6}}{36000}-\frac{5 \eta^{3}}{36}+\frac{\eta^{4}}{144}-\frac{\eta^{7}}{50400}\right)\right) \\
+3 c_{4} \operatorname{PrEc}\left(\frac{1-2 \beta f_{0}^{\prime \prime}(0)+\left(\beta f_{0}^{\prime \prime}(0)\right)^{2}}{6+8 R}\left(\frac{\left(1+c_{1}\right)(3+4 R)}{3 c_{4}}\left(\frac{\eta^{2}}{100}-1\right)\right)\right)
\end{array}\right) \\
+\left(\left(5-\frac{\beta f_{0}^{\prime \prime}(0)}{2}\right)\left(\frac{\eta^{5}}{100000}-\frac{\eta^{4}}{12000}-\frac{1}{6}\right)+\frac{\beta f_{0}^{\prime \prime}(0)}{60}\left(\frac{\eta^{6}}{10}-\eta^{3}\right)\right)+s\left(\frac{\eta^{3}}{300}-\frac{\eta^{2}}{20}+\frac{5}{3}\right)+\lambda\left(1-\beta f_{0}^{\prime \prime}(0)\right)\left(\frac{\eta^{4}}{1200}-\frac{\eta^{5}}{20000}-\frac{10}{3}\right) \\
+\lambda \beta f_{0}^{\prime \prime}(0)\left(\frac{\eta^{3}}{60}-\frac{\eta^{4}}{1200}-\frac{25}{3}\right)
\end{array}\right] \\
& \frac{-3 c_{5}\left(1+\gamma \theta_{0}^{\prime}\right)}{3+4 R}\left(\left(\frac{1}{2}-\frac{1}{2} \beta f_{0}^{\prime \prime}(0)+\lambda\left(\beta f_{0}^{\prime \prime}(0)-1\right)\right)\left(\frac{\eta^{4}}{1200}-\frac{25}{3}\right)-\left(\beta f_{0}^{\prime \prime}(0)+\lambda\left(1-2 \beta f_{0}^{\prime \prime}(0)\right)\right)\left(\frac{\eta^{3}}{60}-\frac{50}{3}\right)+\frac{\left(s+10 \lambda \beta f_{0}^{\prime \prime}(0)\right)}{2}\left(\frac{\eta^{2}}{10}-10\right)\right) \\
& +\frac{3 c_{\text {SPF }} \mathrm{EC}}{3+4 R}\left(\left(\beta f_{0}^{\prime \prime}(0)\right)^{2}-2 \beta f_{0}^{\prime \prime}(0)+1\right)\left(\frac{\eta^{2}}{10}-5 \eta\right) \text {. }
\end{aligned}
$$


The third-order problem is

$$
\left\{\begin{array}{l}
\left.f_{3}^{\prime \prime}\left(\eta, c_{1}, c_{2}, c_{3}\right)\right)\left(1+c_{1}\right) f_{2}^{\prime \prime}+c_{2} f_{1}^{\prime \prime}+\frac{c_{3}}{\operatorname{Pr}}\left(f_{0} f_{0}^{\prime \prime}-f_{0}^{\prime 2}+\left(\Omega+M^{2}\right)\left(1-f_{0}^{\prime}\right)\right) \\
+\frac{c_{2}}{\operatorname{Pr}}\left(f_{0} f_{1}^{\prime \prime}+f_{1} f_{0}^{\prime \prime}-2 f_{0}^{\prime} f_{1}^{\prime}-f_{1}^{\prime}\left(\Omega+M^{2}\right)\right)-\frac{c_{1}}{\operatorname{Pr}}\left[2 f_{0}^{\prime} f_{2}^{\prime}+f_{1}^{2}+f_{2}^{\prime}\left(\Omega+M^{2}\right)\right], \\
\theta_{3}^{\prime \prime}\left(\eta, c_{4}, c_{5}, c_{6}\right)=\left(1+c_{4}\right) \theta_{2}^{\prime \prime}+c_{5} \theta_{1}^{\prime \prime}+\frac{3 c_{4}}{3+4 R}\left[\begin{array}{c}
\left.f_{0} \theta_{2}^{\prime}+f_{1} \theta_{1}^{\prime}+f_{2} \theta_{0}^{\prime}-\lambda\left(f_{0}^{\prime} \theta_{2}+f_{1}^{\prime} \theta_{1}+f_{2}^{\prime} \theta_{0}\right)\right] \\
+\operatorname{PrEc}\left(2 f_{0}^{\prime \prime} f_{2}^{\prime \prime}+f_{1}^{, 2}\right) \\
+\frac{3 c_{5}}{3+4 R}\left(f_{1} \theta_{0}^{\prime}+f_{0} \theta_{1}^{\prime}-\lambda\left(f_{1}^{\prime} \theta_{0}+f_{0}^{\prime} \theta_{1}\right)+\operatorname{PrEc}\left(2 f_{0}^{\prime \prime} f_{1}^{\prime \prime}\right)\right)-\frac{3 c_{6} \lambda}{3+4 R} f_{0}^{\prime} \theta_{0},
\end{array}\right]
\end{array}\right.
$$

with boundary conditions:

$$
\left\{\begin{array}{l}
f_{3}(0)=0, \\
f_{3}^{\prime}(0)=0, \\
\theta_{3}(0)=0, \\
f_{3}^{\prime}(\eta)=0, \\
\theta_{3}(\eta)=0, \quad \text { as } \eta \longrightarrow \infty .
\end{array}\right.
$$

The solution for equation (38) subject to the boundary conditions in equation (39) is obtained in a manner similar to that in equations (29), (32), and (35). However, its expression is very large to be included in this paper.

Collecting the solution components of equations (31), (34), and (37) and the solution of equation (38), the fourterm solution obtained by OHAM for $p=1$ is

$$
\left\{\begin{array}{l}
\tilde{f}\left(\eta, c_{1}, c_{2}, c_{3}\right)=f_{0}(\eta)+f_{1}\left(\eta, c_{1}\right)+f_{2}\left(\eta, c_{1}, c_{2}\right)+f_{3}\left(\eta, c_{1}, c_{2}, c_{3}\right), \\
\tilde{\theta}\left(\eta, c_{4}, c_{5}, c_{6}\right)=\theta_{0}(\eta)+\theta_{1}\left(\eta, c_{4}\right)+\theta_{2}\left(\eta, c_{4}, c_{5}\right)+\theta_{3}\left(\eta, c_{4}, c_{5}, c_{6}\right) .
\end{array}\right.
$$

To find the value of $c_{i}^{\prime} s, i=1,2, \ldots, 6$ in equation (40) for $\operatorname{Pr}=0.72, \beta=\gamma=0.2, R=\Omega=M=s=\lambda=1$, and $\mathrm{Ec}=0.1$, we applied the least-square method to decide the values of convergence control parameters and we get $c_{1}=$ $-0.0137964 ; c_{2}=4.181133 ; c_{3}=-0.006856 ; c_{4}=-0.04417926$; $c_{5}=-9.05104899 ; c_{6}=2.236272$.

By substituting these values into equation (40), we obtain the solution for the problem.

\section{Results and Discussion}

In this work, we employed OHAM to develop the convergent solutions for nonlinear system equations (22) and (25) subject to the BCs equations (23) and (26). Analyses of the effects of various embedded parameters in the flow model on temperature, momentum of the fluid, skin friction coefficient, and local Nusselt number have been made, and the fallouts of the investigative computations are presented via tables and different figures and are discussed.

5.1. Comparative Analysis. To confirm the accuracy and appropriateness of the method, we have compared the value of the skin friction coefficient $f^{\prime \prime}(0)$ and the temperature ramp at the wall $-\theta^{\prime}(0)$ obtained by the present method with the results reported by previous studies under limiting conditions [35-37]. The comparisons of the obtained outcome are presented in Tables 1 and 2 , illustrating an excellent agreement, and thus we are confident that the method is suitable for investigation of the problem. Table 1 is prepared to show an association of the discrepancy of the friction coefficient between a fluid and its enclosing surface for some values of suction/blowing and magnetic meadow parameters with previous studies. From the table, it is observed that our result is in excellent agreement with the results reported previously by Bana et al. [37], Bhatti et al. [35], and Kechil and Hashim [36]. The table also reveals that local skin friction coefficient $f^{\prime \prime}(0)$ increases with growing values of $s$ and $M$.

In Table 2, a relationship of the change in local Nusselt number on behalf of numerous values of suction/blowing parameter and magnetic flux parameter with previously reported data in the cited literature was presented. As is illustrated in Table 2, the results are in good agreement with the results reported by Bana et al. [37] and Bhatti et al. [35]. From the table, it is also obvious that the Nusselt number rises with the charismatic field parameter $(M)$ value. 
TABLE 1: Comparison of the values of local skin friction coefficient $\left(f^{\prime \prime}(0)\right)$ obtained by the OHAM and other numerical methods in the existing literature for altered values of $M$ and $s$ when $\operatorname{Pr}=1, \beta=\Omega=0$.

\begin{tabular}{cccccc}
\hline$s$ & $M$ & Present result & Bana et al. [37] & Bhatti et al. [35] & Kechil and Hashim [36] \\
\hline & 0 & 0.756568 & 0.756575 & 0.7565 & 0.7559794662 \\
-1 & 1 & 1.116430 & 1.116421 & 1.1164 & 1.1164292350 \\
& 2 & 1.877623 & 1.877621 & 1.8776 & 1.8776221492 \\
& 5 & 4.667523 & 4.667525 & - & 4.6675255187 \\
& 10 & 9.585915 & 9.585914 & - & 9.5859131993 \\
\hline & 0 & 1.232564 & 1.232586 & 1.2325 & 1.230652493 \\
& 1 & 1.585312 & 1.585331 & 1.5853 & 2.5852039585 \\
& 2 & 2.346640 & 2.346663 & - & 5.1476635110 \\
& 5 & 5.147941 & 5.14793 & - & 10.074741096 \\
\hline & 10 & 10.07473 & 10.074742 & 1.8893 & 10.074741096 \\
& 0 & 1.889322 & 1.889312 & 2.2029 & 2.2014335458 \\
& 1 & 2.202917 & 2.202933 & 2.92029 & 2.9201142028 \\
& 2 & 2.920113 & 2.920110 & - & 5.6768303421 \\
& 5 & 5.676831 & 5.676830 & - & 10.5883674767 \\
\hline
\end{tabular}

TABle 2: Comparison of values of the local Nusselt number $\left(-\theta^{\prime}(0)\right)$ obtained by the present method and other numerical methods in the existing literature for different $s$ and $M$ values when $\operatorname{Pr}=1, \beta=\Omega=\lambda=0$.

\begin{tabular}{ccccc}
\hline$s$ & $M$ & Present result & Bana et al. [37] & Bhatti et al. [35] \\
\hline \multirow{3}{*}{-1} & 0 & 0.11676 & 0.11675 & 0.11677 \\
& 1 & 0.14004 & 0.1400 & 0.14000 \\
& 2 & 0.17313 & 0.17312 & 0.17312 \\
\hline \multirow{3}{*}{0} & 0 & 0.57051 & 0.57046 & 0.57035 \\
& 1 & 0.59537 & 0.59535 & 0.59539 \\
& 2 & 0.63416 & 0.63413 & 0.63418 \\
\hline \multirow{3}{*}{1} & 0 & 1.32369 & 1.32368 & 1.32368 \\
& 1 & 1.33805 & 1.33804 & 1.33804 \\
& 2 & 1.36447 & 1.36446 & 1.36446 \\
\hline
\end{tabular}

5.2. Physical Description. The optimal homotopy analysis method described in Section 2 of this paper is employed to find the solution for nonlinear differential system. Here, we will discuss the impact of relevant parameters in the flow model on velocity and temperature distribution.

5.3. Velocity. The influence of induced magnetic field, permeability parameter, velocity slip parameter, suction/ blowing parameter, and Eckert number on velocity profile is illustrated in Figures 2-6.

Figure 2 illustrates the magnetic field effects on the fluid velocity distribution. It is detected from the figure that boundary layer thickness and the fluid velocity profile decline against the charismatic field $M$. This result is manifested from reality, because the presence of a magnetic field produces a body force, called Lorentz force, which opposes the fluid motion and, hence, it reduces the fluid freedom of movement. Therefore, when the magnetic flux increases, the retardation force also increases, and this resistance offered to the flow is responsible for decreasing the fluid velocity. Figure 3 demonstrates the effect of penetrability parameter on the fluid velocity dispersal. It has been realized that the rising of the penetrability parameter value results in

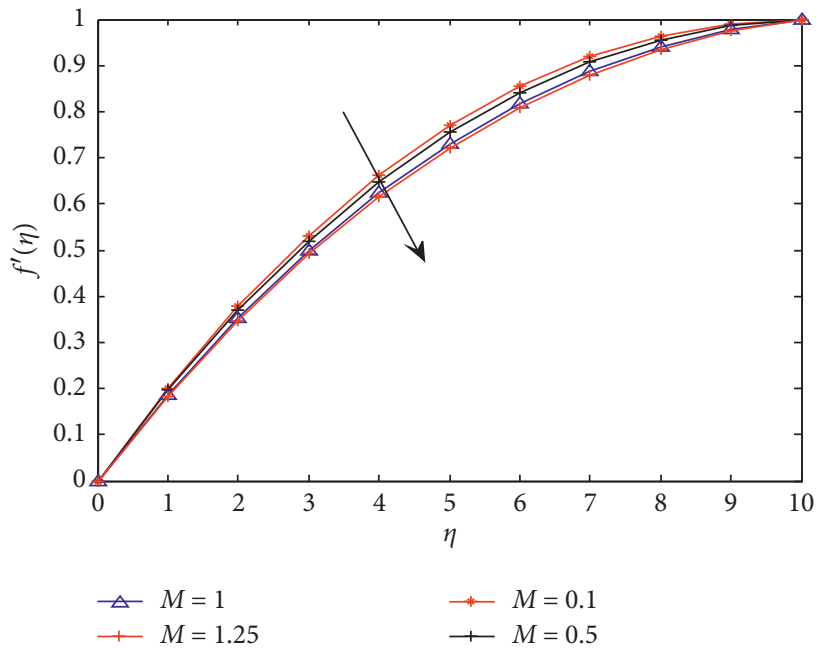

Figure 2: Velocity profile for numerous values of $M$ when $\operatorname{Pr}=0.72, \Omega=\lambda=R=s=1, \beta=0.2, \gamma=0.2$, and $\mathrm{Ec}=0.1$.

increasing the velocity profile, which causes an increment in the width of the velocity boundary wall layer. Physically, it is clear that porous medium reduces the motion of the fluid. Thus, as the permeability parameter $\Omega$ increases resistance to the motion of the fluid along the surface drops, in other words, when the value of porosity parameter $K$ declines, the velocity boosts with the increase of the value of $\Omega$.

Figure 4 exhibits the graphical illustration of the impact of velocity slip parameter $\beta$ on momentum (velocity) profiles. From the figure, it is seen that intensification in slip parameter results in deterioration of velocity profile. This is due to the fact that an upsurge in the velocity slip factor produces friction force, which allows more fluid to slip past the sheet, and consequently the motion of the flow decelerates. As it is exhibited by Figure 5, the momentum profile was raised with suction/blowing parameter $s$. In fact, the action of suction increases the momentum boundary layer thickness, which results in accelerating the motion of the fluid. The alteration of value of the ratio of the advective 


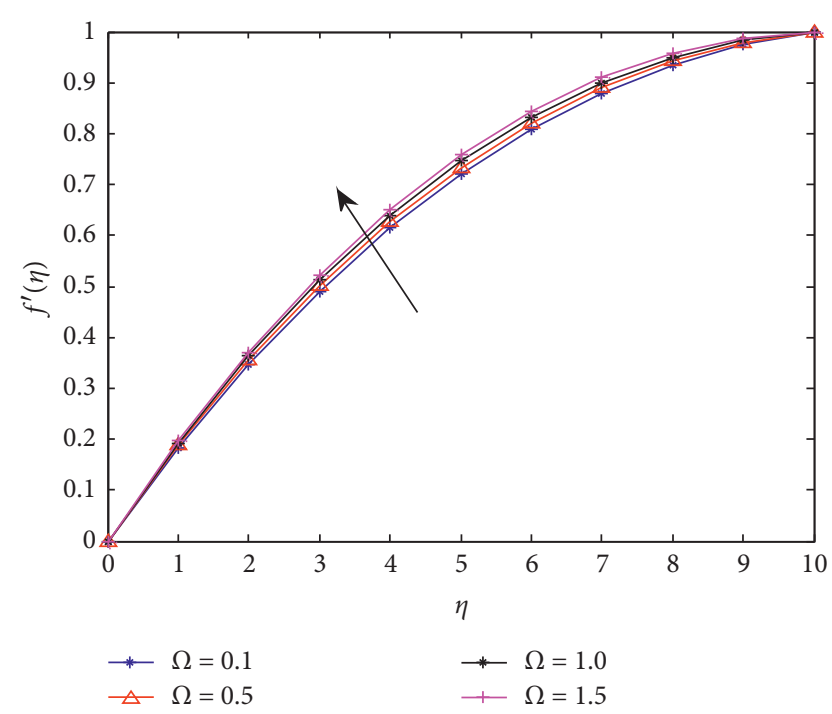

Figure 3: Velocity profile for various values of $\Omega$ when $\operatorname{Pr}=0.72$, $M=\lambda=R=s=1, \beta=0.2, \gamma=0.2$, and $\mathrm{Ec}=0.1$.

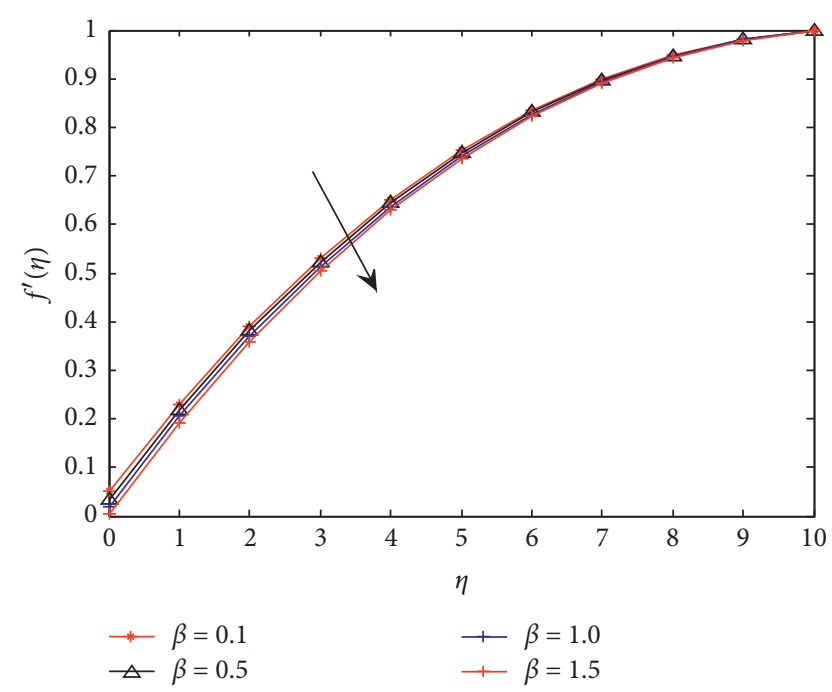

FIGURE 4: Velocity profile for altered values of $\beta$ when $\operatorname{Pr}=0.72$, $\Omega=M=\lambda=R=s=1, \gamma=0.2$, and $\mathrm{Ec}=0.1$.

mass transfer to the heat dissipation potential Ec does not affect velocity of the fluid as is displayed in Figure 6. Likewise, altering radiation parameter, wall temperature exponent, and thermal slip values does not disturb the momentum profile of the fluid in motion; this is evident from the fact that the velocity equation is not being coupled with the energy equation of the problem.

5.4. Temperature. The impact of Prandtl number, suction/ blowing parameter, permeability parameter, velocity slip parameter, radiation, Eckert number, wall temperature parameter, thermal slip parameter, and magnetic field parameter on temperature distribution is displayed in Figures 7-14. Figure 7 shows that the fluid temperature is reducing contrary to Prandtl number Pr. This implies that the thermal boundary layer viscosity diminishes in

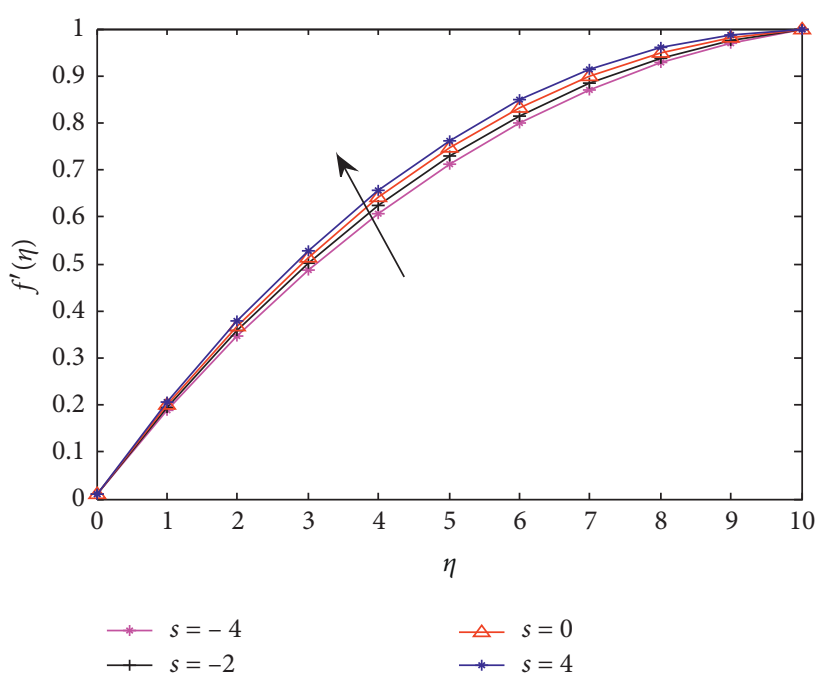

FIgURE 5: Velocity sketch for diverse values of $s$ when $\operatorname{Pr}=0.72$, $\Omega=M=\lambda=R=1, \beta=0.2, \gamma=0.2$, and $\mathrm{Ec}=0.1$.

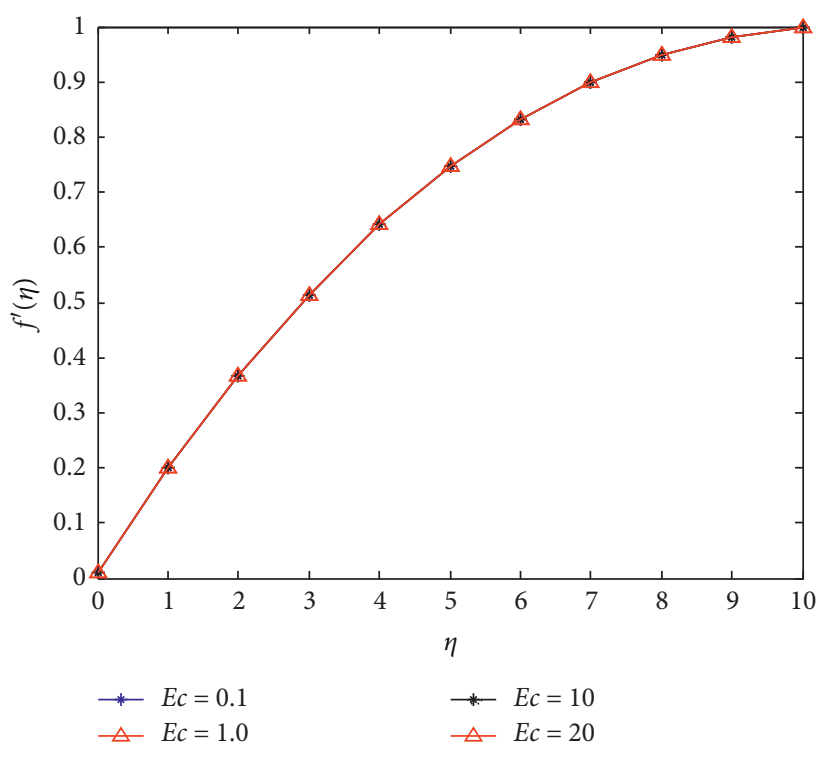

FIgURE 6: Velocity graph for altered values of $\mathrm{Ec}$ when $\operatorname{Pr}=0.72$, $\Omega=M=\lambda=R=s=1, \gamma=0.2$, and $\mathrm{Ec}=0.1$.

opposition to the value of Pr. This happens basically because high value of Prandtl number relates to weak thermal diffusivity, which upshots in a thinner thermal boundary layer. As a matter of fact, intensification in the Prandtl number infers an upsurge in fluid viscosity, which consecutively causes deterioration in temperature distribution. Hence, $\mathrm{Pr}$ can be used to upturn the rate of cooling in flows. Figure 8 displays the alteration in temperature pertaining to the suction/blowing parameter $s$. It is noticed from the figure that an upsurge in the suction/blowing parameter value results in decreasing the heat transmission proportion from the wall. For the reason that, with strong blowing, the thermal boundary thickness increases and accordingly the heated fluid moves farther from the wall and forms an insulating layer of nearly the equivalent temperature as that of 


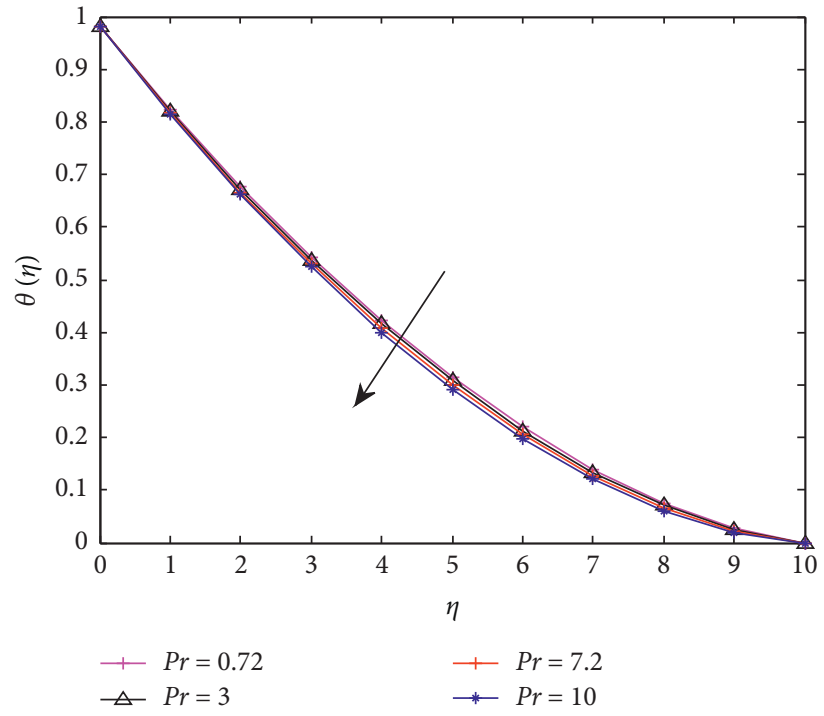

Figure 7: Temperature distribution for numerous values of $\operatorname{Pr}$ when $\Omega=M=\lambda=R=s=1, \gamma=0.2, \beta=0.2$, and Ec $=0.1$.

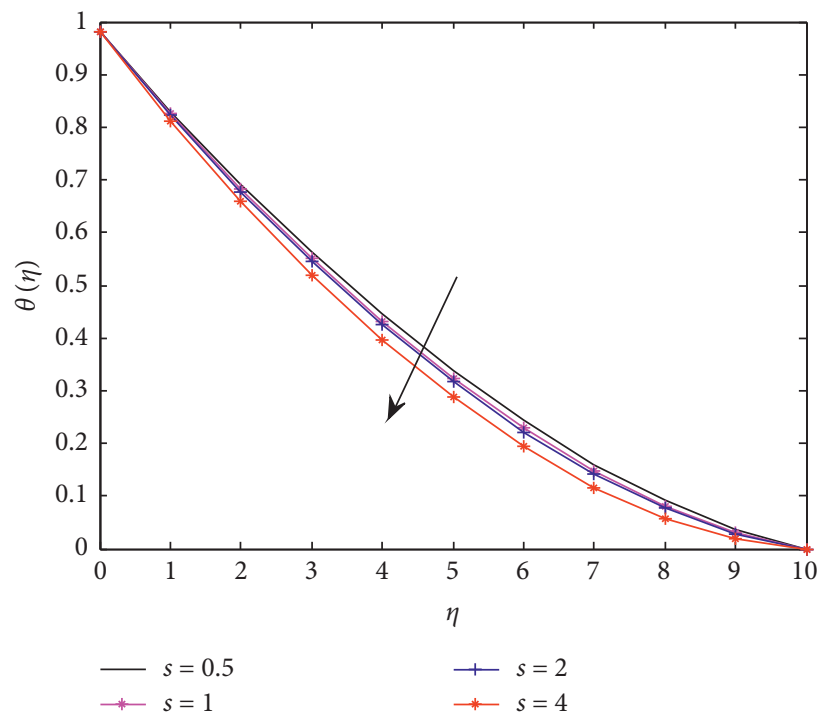

FIGURE 8: Variation of temperature against various values of $s$ when $\operatorname{Pr}=0.72, \Omega=M=\lambda=R=1, \gamma=0.2, \beta=0.2$, and Ec $=0.1$.

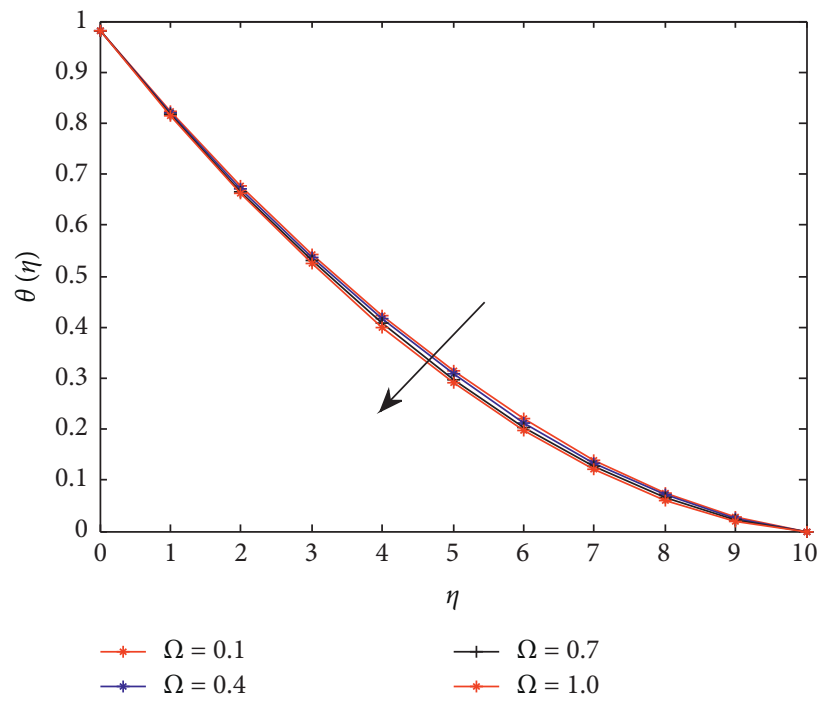

FIgURE 9: Variation in temperature against various values of $\Omega$ when $\operatorname{Pr}=0.72, M=\lambda=R=s=1, \gamma=0.2, \beta=0.2$, and Ec $=0.1$. 


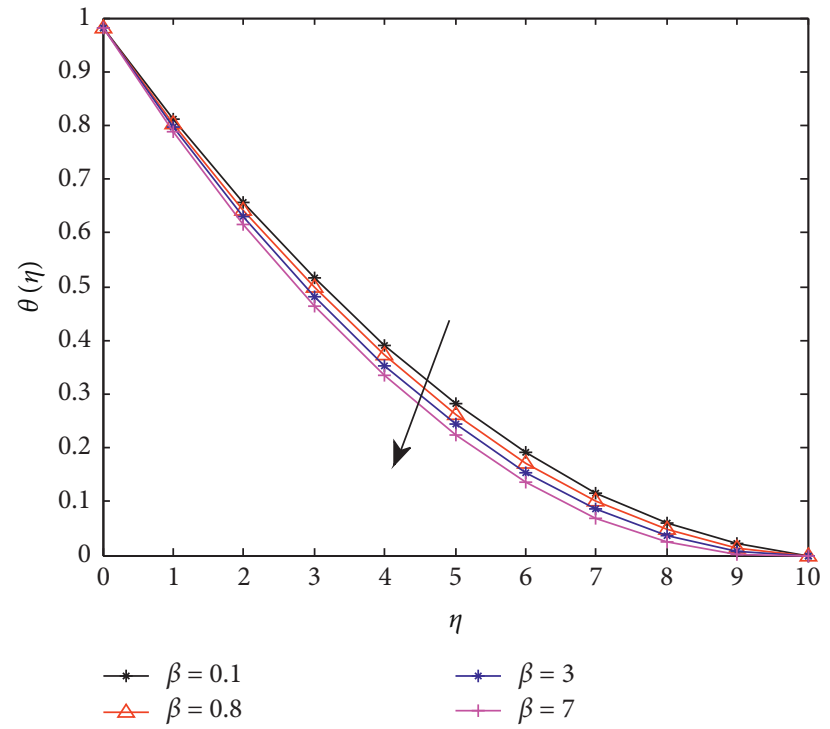

Figure 10: Temperature alteration for numerous values of $\beta$ when $\operatorname{Pr}=0.72, \gamma=0.2, \Omega=M=\lambda=R=s=1, \gamma=0.2$, and Ec $=0.1$.

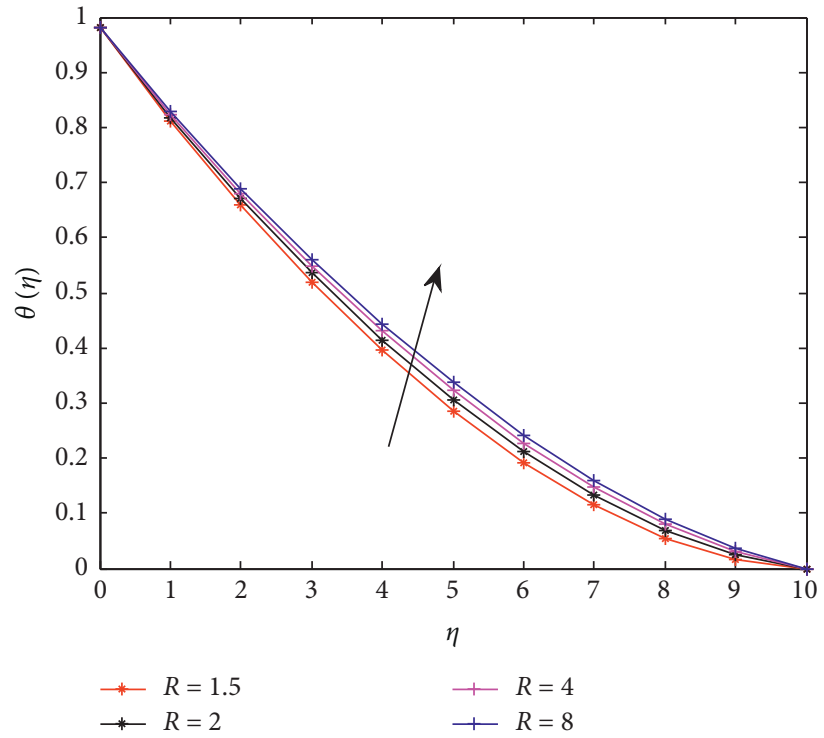

FIGURE 11: Alteration in temperature for various values of $R$ when $\operatorname{Pr}=0.72, \beta=0.2, \Omega=M=\lambda=R=s=1, \gamma=0.2$, and Ec $=0.1$.

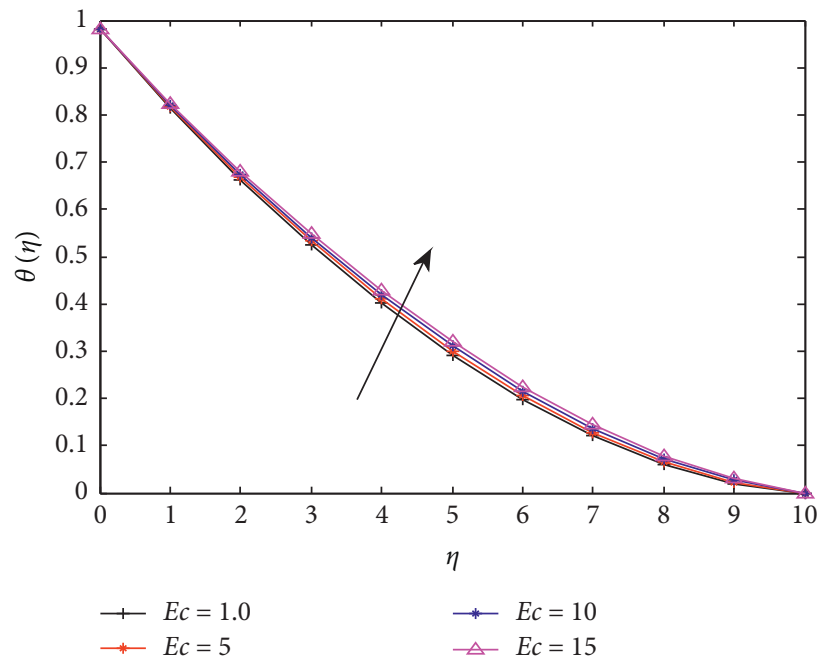

Figure 12: Temperature profile for altered values of Ec when $\operatorname{Pr}=0.72, \beta=0.2, \Omega=M=\lambda=R=s=1$, and $\gamma=0.2$. 


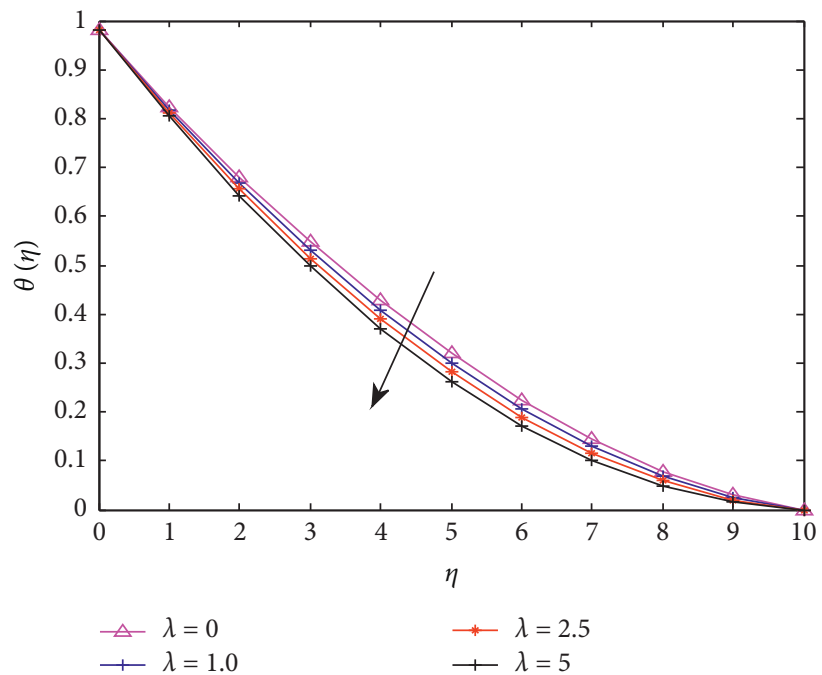

FiguRE 13: Temperature sketch for altered values of $\lambda$ when $\operatorname{Pr}=0.72, \Omega=M=R=s=1, \beta=0.2, \gamma=0.2$, and $\mathrm{Ec}=0.1$.

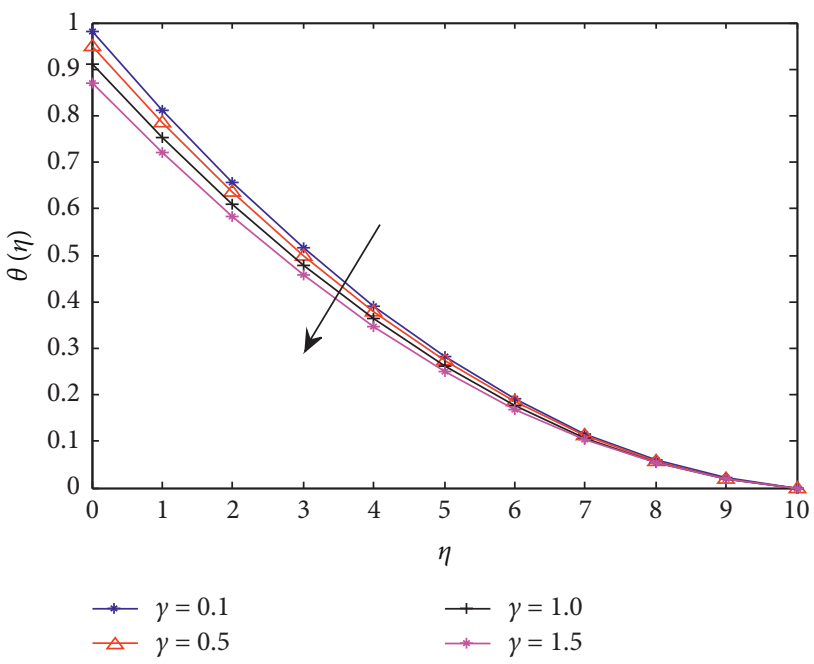

FIgURE 14: Temperature sketch for various values of $\gamma$ on when $\operatorname{Pr}=0.72, \Omega=M=\lambda=R=s=1, \beta=0.2$, and $\mathrm{Ec}=0.1$.

the wall, suction can be used as a means for freezing a surface.

Figure 9 describes the impact of penetrability of the medium on temperature, and it reveals that the thermal boundary layer thickness decreases on account of an increase in the value of penetrability parameter $\Omega$. Physically, permeability of porous medium increases means porosity of the medium, opposing the fluid motion, which decreases. This reduction in resistance to the flow is responsible for offering a decrease in the temperature. Figure 10 represents the temperature sketch on behalf of numerous input values of velocity slip parameter $\beta$, and it reveals that the thermal boundary layer thicknesses declines with increased value of $\beta$. Therefore, stronger unrestricted velocity results in reduction of the temperature and the width of thermal boundary layer. In Figure 11, the effect of radiation $R$ on temperature of the fluid was illustrated and the figure displayed that the thermal boundary layer width and the

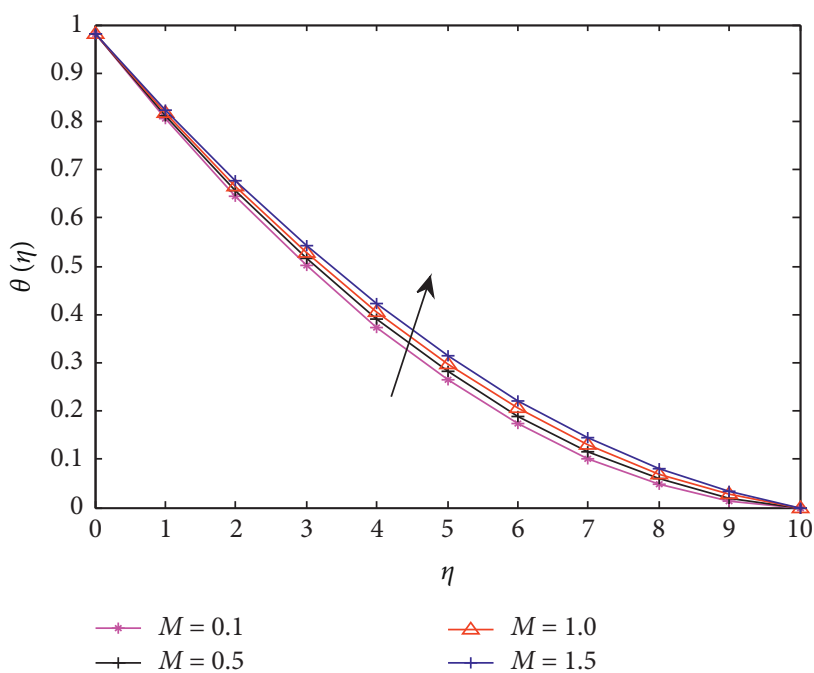

Figure 15: Temperature sketch for altered values of $M$ when $\operatorname{Pr}=0.72, \gamma=0.2, \Omega=\lambda=R=s=1, \beta=0.2$, and $\mathrm{Ec}=0.1$.

temperature dissemination rise as $R$ increases. This happens since the divergence of the radiative heat change increases with the decreasing value of the Rosseland radiative absorptivity. This, in turn, leads to an upsurge in the rate of radiative heat transmission to the fluid, which in turn enhances the fluid temperature.

The distinction in the fluid temperature owing to the alteration of Eckert number Ec has been illustrated in Figure 12. The figure shows that, with increased value of Ec, the fluid temperature intensifies. This happens because heat is produced in the fluid as the value of Ec increases due to frictional heating. Physically, Eckert number is the ratio of kinetic energy to the specific enthalpy difference between wall and fluid. Therefore, an increase in Eckert number causes the transformation of kinetic energy into internal energy by work that is done against the viscous fluid stresses. Due to this, increasing Ec enhances the temperature of the 
fluid. In Figure 13, the impact of wall temperature exponent $\lambda$ on the layer of a liquid between the free stream and a heatexchange surface is displayed, and it has been detected that the fluid temperature decreases with the increasing value of $\lambda$.

The effect of thermal slip $\gamma$ on temperature of the fluid was displayed in Figure 14. It is detected that the temperature dispersion within the fluid in flow is decreased when the parameter that denotes thermal slip gradually upsurges in value. Physically, this is evident from the point that, following in increasing thermal slip, less heat is shifted from the boundary to the fluid, which is responsible for the temperature distribution reduction. In Figure 15, the influence of magnetic field $M$ on the temperature profile of the fluid has been displayed. From the figure, it is noticeable that the temperature boosts with rise in the magnetic field parameter $M$. Physically, when $M$ is increased, an opposing force (Lorentz force) that retards the flow motion will be produced in the fluid. In fact, the presence of magnetic field decelerates velocity and consecutively persuades the temperature field that increases the temperature profiles.

\section{Conclusion}

In this work, magnetohydrodynamic Hiemenz boundary layer flow over a plate with the stagnation area considered against a smooth plate through a holey medium with thermal and velocity slip effects is considered. The liquid in flow is assumed to be electrically conducted and incompressible. A continuous magnetic field is applied along the vertical direction, the wall temperature is taken as variable, and the surface mass fluctuation is supposed to be uniform. The Hall effects and the internal friction terms are disused. The nonlinear equation describing the flow problem is converted to third-order nonlinear ODEs via similarity variable transformation system and then solved by the use of the OHAM. The effects of various parameters in the model of the problem on temperature and velocity are analysed. In order to confirm the applicability and accuracy of the method, the obtained outcomes are verified with some successful and available theoretical data in the literature [35-37] and the comparative outcomes showed an excellent agreement with the current computational analysis.

From the findings of the study, the following conclusions were drawn:

(1) The thickness of the velocity boundary layer and the profile of the fluid velocity decreases as $M$ (the magnetic field) increases; however, the temperature increased as the charismatic field parameter $(M)$ increased in value.

(2) The fluid velocity rises with the increased values of both the penetrability parameter $(\Omega)$ and suction/ blowing parameter $(s)$.

(3) The flow movement, the thermal boundary layer, and gradient of the temperature reduce against the velocity slip parameter $(\beta)$.
(4) The temperature of the fluid rises in conjunction with the Eckert number $(\mathrm{Ec})$ but the velocity of the fluid is not influenced by varying the value of Ec.

(5) An upsurge in thermal slip parameter results in a discount in the thermal boundary layer thickness and so reduces the temperature profile.

(6) The thermal boundary layer thickness upturns following an increased value of both the magnetic field parameter and radiation parameter, but it declines in conjunction with an increased value of Prandtl number $(\operatorname{Pr})$ and the exponent of wall temperature parameter $(\lambda)$.

(7) The skin friction coefficient and the Nusselt number increase with rising value of suction/blowing and magnetic field parameter.

\section{Data Availability}

The data used to support the findings of this investigation are encompassed in this article.

\section{Conflicts of Interest}

The authors declare that there are no conflicts of interest regarding the publication of this paper.

\section{Acknowledgments}

The authors express their heartfelt gratitude to the authors of literature studies used in this paper for the provided scientific aspects and ideas that supplement this work.

\section{References}

[1] K. Hiemenz, "Die Grenzschicht an einem in Den Gleichformigen Flussigkeitsstrom eingetauchten geraden Kreiszylinder," International Journal of Scientific \& Technology Research, vol. 326, pp. 321-324, 1911.

[2] A. Ishak, K. Jafar, R. Nazar, and I. Pop, "MHD stagnation point flow towards a stretching sheet," Physica A: Statistical Mechanics and Its Applications, vol. 388, no. 17, pp. 33773383, 2009.

[3] T. R. Mahapatra, S. K. Nandy, and A. S. Gupta, "Momentum and heat transfer in MHD stagnation-point flow over a shrinking sheet," Journal of Applied Mechanics, vol. 78, 2011.

[4] D. S. Anjali and D. R. Uma, "Effects of thermal radiation on hydromagnetic flow due to a porous rotating disk with hall effect," Journal of Applied Fluid Mechanics, vol. 5, no. 2, pp. 1-7, 2012.

[5] A. J. Chamkha and S. E. Ahmed, "Similarity solution for unsteady MHD flow near a stagnation point of a three-dimensional porous body with heat and mass transfer," Heat Generation/absorption and Chemical Reaction, vol. 4, no. 2, pp. 87-94, 2011.

[6] K. Bhattacharyya, S. Mukhopadhyay, and G. C. Layek, "Slip effects on boundary layer stagnation-point flow and heat transfer towards a shrinking sheet," International Journal of Heat and Mass Transfer, vol. 54, no. 1-3, pp. 308-313, 2011.

[7] T. R. Mahapatra and A. S. Gupta, "Magnetohydrodynamic stagnation-point flow towards a stretching sheet," Acta Mechanica, vol. 152, no. 1-4, pp. 191-196, 2001. 
[8] T. Hayat, Z. Abbas, M. Sajid, and S. Asghar, "The influence of thermal radiation on MHD flow of a second grade fluid," International Journal of Heat and Mass Transfer, vol. 50, no. 56, pp. 931-941, 2007.

[9] O. D. Makinde, W. A. Khan, and Z. H. Khan, "Buoyancy effects on MHD stagnation point flow and heat transfer of a nanofluid past a convectively heated stretching/shrinking sheet," International Journal of Heat and Mass Transfer, vol. 62, pp. 526-533, 2013.

[10] S. Qayyum, T. Hayat, S. A. Shehzad, and A. Alsaedi, "Effect of a chemical reaction on magnetohydrodynamic (MHD) stagnation point flow of Walters-B nanofluid with Newtonian heat and mass conditions," Nuclear Engineering and Technology, vol. 49, no. 8, pp. 1636-1644, 2017.

[11] I. Husain, F. Labropulu, and I. Pop, "Two-dimensional oblique stagnation-point flow towards a stretching surface in a viscoelastic fluid," Central European Journal of Physics, vol. 9, no. 1, pp. 176-182, 2011.

[12] S. S. Motsa and S. Shateyi, "A new approach for the solution of three-dimensional magnetohydrodynamic rotating flow over a shrinking sheet," Mathematical Problems in Engineering, vol. 2010, Article ID 586340, 15 pages, 2010.

[13] M. Shen, F. Wang, and H. Chen, "MHD mixed convection slip flow near a stagnation-point on a nonlinearly vertical stretching sheet," Boundary Value Problems, vol. 1, p. 78, 2015.

[14] P. R. Sharma, S. Sinha, R. S. Yadav, and A. N. Filippov, "MHD mixed convective stagnation point flow along a vertical stretching sheet with heat source/sink," International Journal of Heat and Mass Transfer, vol. 117, pp. 780-786, 2018.

[15] M. M. Bhatti and M. M. Rashidi, "Numerical simulation of entropy generation on MHD nanofluid towards a stagnation point flow over a stretching surface," International Journal of Applied and Computational Mathematics, vol. 3, no. 3, pp. 2275-2289, 2017.

[16] M. Sajid and T. Hayat, "Influence of thermal radiation on the boundary layer flow due to an exponentially stretching sheet," International Communications in Heat and Mass Transfer, vol. 35, no. 3, pp. 347-356, 2008.

[17] S. Mukhopadhyay and R. S. R. Gorla, "Effects of partial slip on boundary layer flow past a permeable exponential stretching sheet in presence of thermal radiation," Heat and Mass Transfer, vol. 48, no. 10, pp. 1773-1781, 2012.

[18] M. I. Khan and A. Faris, "Dynamics of activation energy and nonlinear mixed convection in Darcy-forchheimer radiated flow of carreau nanofluid near stagnation point region," Journal of Thermal Science and Engineering Applications, vol. 13, no. 5, 2021.

[19] S. Chaudhary, S. Chaudhary, and C. Susheela, "Thermal radiation effects on MHD boundary layer flow over an exponentially stretching surface," Applied Mathematics, vol. 06, no. 02, p. 295, 2015.

[20] O. D. Makinde, "Heat and mass transfer by MHD mixed convection stagnation point flow toward a vertical plate embedded in a highly porous medium with radiation and internal heat generation," Meccanica, vol. 47, no. 5, pp. 1173-1184, 2012.

[21] I. Wubshet, "Magnetohydrodynamic (MHD) stagnation point flow and heat transfer of upper-convected Maxwell fluid past a stretching sheet in the presence of nanoparticles with convective heating," Frontiers in Heat and Mass Transfer (FHMT), vol. 7, no. 1, 2016.

[22] T. R. Mahapatra and S. K. Nandy, Momentum and Heat Transfer in MHD Axisymmetric Stagnation-point Flow over a Shrinking Sheet, 2013.
[23] M. I. Khan, M. Hayat, T. H. Alsaedi, and A. Ahmed, "A comparative study of Casson fluid with homogeneousheterogeneous reactions," Journal of Colloid and Interface Science, vol. 498, pp. 85-90, 2017.

[24] S. Shateyi and F. Mabood, "MHD mixed convection slip flow near a stagnation-point on a non-linearly vertical stretching sheet in the presence of viscous dissipation," Thermal Science, vol. 21, no. 6, pp. 2731-2745, 2017.

[25] M. I. Khan and A. Faris, "Cattaneo-Christov Double Diffusion (CCDD) and magnetized stagnation point flow of nonNewtonian fluid with internal resistance of particles," Physica Scripta, vol. 95, no. 12, 2020.

[26] D. S. Anjali and D. R. Uma, Effects of thermal radiation on hydromagnetic flow due to a porous rotating disk with hall effect, 2012.

[27] M. I. Khan and F. Alzahrani, "Nonlinear dissipative slip flow of Jeffrey nanomaterial towards a curved surface with entropy generation and activation energy," Mathematics and Computers in Simulation, vol. 185, pp. 47-61, 2021.

[28] M. I. Khan and F. Alzahrani, "Free convection and radiation effects in nanofluid (silicon dioxide and molybdenum disulfide) with second order velocity slip, entropy generation, Darcy-Forchheimer porous medium," International Journal of Hydrogen Energy, vol. 46, no. 1, pp. 1362-1369, 2021.

[29] M. I. Khan, "Transportation of hybrid nanoparticles in forced convective Darcy-Forchheimer flow by a rotating disk," International Communications in Heat and Mass Transfer, vol. 122, Article ID 105177, 2021.

[30] T. Fang, J. Zhang, and S. Yao, "Slip magnetohydrodynamic viscous flow over a permeable shrinking sheet," Chinese Physics Letters, vol. 27, no. 12, Article ID 124702, 2010.

[31] El Aziz and A. A. Ahmed, "Influences of slip velocity and induced magnetic field on MHD stagnation-point flow and heat transfer of Casson fluid over a stretching sheet," Mathematical Problems in Engineering, vol. 2018, no. 11, 2018.

[32] V. Marinca and N. Herişanu, "Determination of periodic solutions for the motion of a particle on a rotating parabola by means of the optimal homotopy asymptotic method," Journal of Sound and Vibration, vol. 329, no. 9, pp. 1450-1459, 2010.

[33] B. Solomon and F. Daba, "Analytical analysis of effects of buoyancy, internal heat generation, magnetic field, and thermal radiation on a boundary layer over a vertical plate with a convective surface boundary condition," International Journal of Differential Equations, vol. 202016 pages, 2020.

[34] A. A. Raptis and H. S. Takhar, "Flow through a porous medium," Mechanics Research Communications, vol. 14, no. 5-6, pp. 327-329, 1987.

[35] M. M. Bhatti and M. M. Rashidi, "Effects of thermo-diffusion and thermal radiation on Williamson nanofluid over a porous shrinking/stretching sheet," Journal of Molecular Liquids, vol. 221, pp. 567-573, 2016.

[36] S. A. Kechil and I. Hashim, "Approximate analytical solution for MHD stagnation-point flow in porous media," Communications in Nonlinear Science and Numerical Simulation, vol. 14, no. 4, pp. 1346-1354, 2009.

[37] N. Bano, B. B. Singh, and S. R. Sayyed, "Homotopy analysis for MHD hiemenz flow in a porous medium with thermal radiation, velocity and thermal slips effects," Frontiers in Heat and Mass Transfer (FHMT), vol. 10, 2018. 